\title{
Orthostatic and Supine Blood Pressures Are Associated with White Matter Hyperintensities in Parkinson Disease
}

\author{
Yoon-Sang Oh \\ Joong-Seok Kim \\ Kwang-Soo Lee \\ Department of Neurology, \\ College of Medicine, \\ The Catholic University of Korea, \\ Seoul, Korea
}

Received July 12, 2012

Revised June 6, 2013

Accepted July 5, 2013

\section{Corresponding author}

Joong-Seok Kim, MD, PhD

Department of Neurology,

College of Medicine,

The Catholic University of Korea,

Seoul St. Mary's Hospital,

222 Banpo-daero, Seocho-gu,

Seoul 137-701, Korea

Tel $+82-2-2258-6078$

Fax +82-2-599-9686

E-mail neuronet@catholic.ac.kr

- The authors have no financial conflicts of interest.
Background and Purpose: Several reports on the elderly population have suggested that orthostatic hypotension is associated with white matter hyperintensities (WMH); however, little information is available on patients with Parkinson's disease (PD). Methods: We analyzed the association blood pressure profiles during tilt table testing with WMH scores in 117 patients with PD. WMH were rated using the semiquantitative visual rating system proposed by Scheltens et al. Results: The presence of orthostatic hypotension was associated with increasing tendency of WMH score and the blood pressure changes during tilting and supine blood pressure were positively correlated with increasing WMH score. Conclusions: This finding indicates that hemodynamic changes associated with orthostatic hypotension may be associated with white matter changes in patients with PD.

Journal of Movement Disorders 2013;6:23-27

Key Words: Parkinson disease, White matter hyperintensity, Orthostatic hypotension, Supine blood pressure.

White matter hyperintensities (WMH) on T2-weighted magnetic resonance imaging (MRI) sequences of the brain are known to be associated with an increased risk of stroke, dementia, and mortality. ${ }^{1}$ It is well documented that $\mathrm{WMH}$ are closely related to vascular risk factors such as increasing age and hypertension in normal elderly and in patients with stroke. ${ }^{2-4}$ However, little information is available in patients with Parkinson's disease (PD) and pilot studies did not demonstrate what factors are associated with WMH in patients with PD, ${ }^{5,6}$

Orthostatic hypotension $(\mathrm{OH})$ is the most common cardiovascular autonomic dysfunction in patients with PD. ${ }^{7-9}$ Previous studies in normal populations have shown that orthostatic hypotension is associated with an increased prevalence of multiple silent cerebral ischemic events and a higher risk for cerebrovascular damages. ${ }^{10}$

Therefore, we hypothesized that blood pressure changes during tilt table testing can contribute to white matter changes attending early PD. We investigated the influence of orthostatic hypotension on WMH in patients with PD using head-up tilt-testing.

\section{Methods}

\section{Patients}

This study was approved by the local ethics committee, and each patient gave informed consent for participation.

This study included 117 newly diagnosed patients. The clinical diagnosis of PD was made according to the UK Brain Bank criteria. ${ }^{11}$ At the time of the study, none of the patients had ever taken medications for PD. All patients underwent a detailed clinical evaluation, including laboratory tests for lipid profiles and serum homocysteine.

At the time of study enrollment, all included patients were evaluated using the Unified Parkinson's Disease Rating Scale (UPDRS) and the modified Hoehn and Yahr staging score. Tilt table testing was performed after discontinuing antihypertensive drugs for 7 days. 


\section{Tilt table testing}

Patients were studied in a temperature-controlled clinical investigation room. Patients fasted the night before the test, except for a small amount of water, if they were thirsty and abstained from drinking alcohol or coffee the day before the study. Continuous electrocardiographic and non-invasive blood pressure monitoring was used in each patient (YM6000, Mediana Tech., San Antonio, TX, USA). After $30 \mathrm{~min}$ of supine rest, the test $\left(20 \mathrm{~min}\right.$ at $\left.60^{\circ}\right)$ was performed using the Manumed Special Tilt1-section (ENRAF NONIUS, Rotterdam, The Netherlands). By consensus, orthostatic hypotension was defined as a fall in blood pressure of at least $20 \mathrm{~mm} \mathrm{Hg}$ systolic pressure and/or $10 \mathrm{~mm} \mathrm{Hg}$ diastolic pressure between 2 and 5 minutes after tilt. ${ }^{12}$

\section{MRI and WMH rating}

All patients enrolled in the study underwent conventional MRI with a 3.0-T system (Magnetom Verio, 3T, Siemens). Axial T2 weighted images, complemented with coronal fluid attenuated recovery images, were used to rate the WMH.

The MR images were rated using the semiquantitative visual rating system of Scheltens et al. ${ }^{13}$ This rating scale provides four sum scores in a semiquantitative way; periventricular hyperintensities (0-6), deep WMH (0-24), basal ganglia hyperintensities (0-30), and infratentorial hyperintensities (0-30). Periventricular hyperintensities were identified as continuous, confluent areas of high signal intensity adjacent to the anterior or posterior horns of the lateral ventricles ("cap") and along the lateral ventricles ("bands"). Absence of lesions was a score of $0, \leq 5 \mathrm{~mm}$ lesions was a score of 1 , and a score of 2 was given for lesions $>5 \mathrm{~mm}$. Hyperintensities $>10 \mathrm{~mm}$ were scored as lobar white matter changes. WMH, located in the deep and subcortical white matter, were separately rated in the frontal, temporal, parietal, and occipital regions according to the previously described criteria. ${ }^{13}$ These rating criteria were also applied to regions of the basal ganglia (caudate, putamen, globus pallidus, thalamus, and internal capsule) and infratentorial regions (cerebellum, midbrain, pons, and medulla).

\section{Data analysis}

The independent sample $t$-test or Pearson's chi-square test was used to compare the differences between the groups categorized by the blood pressure response during orthostasis (non$\mathrm{OH}$ and $\mathrm{OH}$ ). Given the potential influence of several variables on the results of $\mathrm{WMH}$, we also conducted group comparisons with an analysis of covariance (ANCOVA) including age, the presence of hypertension and diabetes, low density lipoprotein (LDL)-cholesterol, and serum homocysteine levels as confounding covariates. Patients were also divided into no to mild and severe WMHs groups based on two extremes by tertile distribution. Their BP results were analyzed by independent sample $t$-tests. The relationship between blood pressure profiles monitored during tilt table testing tilt and $\mathrm{WMH}$ were tested using Spearman's rank correlation coefficients. The statistical analysis was performed with SPSS software version 15.0 (SPSS, Inc., Chicago, IL, USA). A $p<0.05$ was considered significant.

\section{Results}

Among the 117 patients enrolled, 46 were men and 71 were

Table 1. The demographics of the patients with/without orthostatic hypotension

\begin{tabular}{|c|c|c|c|}
\hline & Non-OH $(n=75)$ & $\mathrm{OH}(n=42)$ & $p$ \\
\hline Age (years) & $67.7 \pm 11.1$ & $69.8 \pm 8.0$ & 0.284 \\
\hline No. of men* $(n, \%)$ & $25(33.3)$ & $21(50.0)$ & 0.077 \\
\hline Disease duration (years) & $2.0 \pm 0.9$ & $2.2 \pm 1.0$ & 0.360 \\
\hline Hypertension* $(n, \%)$ & $19(25.3)$ & $10(23.8)$ & 0.855 \\
\hline Diabetes mellitus* (n, \%) & $13(17.3)$ & $11(26.2)$ & 0.255 \\
\hline \multicolumn{4}{|l|}{ Smoking status* $(\mathrm{n}, \%)$} \\
\hline Non-smoker & $69(92.0)$ & $37(88.1)$ & 0.689 \\
\hline Ex-smoker & $4(5.3)$ & $4(9.5)$ & \\
\hline Current smoker & $2(2.7)$ & $1(2.4)$ & \\
\hline \multicolumn{4}{|l|}{ Lipid profiles (mg/dL) } \\
\hline Total cholesterol & $180.5 \pm 32.2$ & $178.0 \pm 39.0$ & 0.713 \\
\hline LDL-cholesterol & $104.7 \pm 27.5$ & $106.9 \pm 31.4$ & 0.699 \\
\hline H\&Y stage & $1.9 \pm 0.8$ & $1.6 \pm 0.6$ & 0.160 \\
\hline \multicolumn{4}{|l|}{ UPDRS } \\
\hline Total & $26.1 \pm 19.5$ & $22.1 \pm 14.3$ & 0.292 \\
\hline Serum homocysteine level, $\mu \mathrm{mol} / \mathrm{L}$ & $10.1 \pm 5.1$ & $10.2 \pm 2.9$ & 0.968 \\
\hline
\end{tabular}

Values represent mean with standard deviation or numbers of patients with percentage in parenthesis. Analyses were performed by independent sample t-test or *the chi-square test. OH: orthostatic hypotension, LDL: low density lipoprotein, H\&Y: Hoehn and Yahr, UPDRS: Unified Parkinson's Disease Rating Scale. 
Table 2. Comparison of white matter hyperintensities of the patients with/without orthostatic hypotension

\begin{tabular}{lcccc}
\hline \multicolumn{1}{c}{ Brain area } & $\begin{array}{c}\text { Non- } \mathrm{OH} \\
(n=75)\end{array}$ & $\begin{array}{c}\mathrm{OH} \\
(n=42)\end{array}$ & $\mathrm{F}$ & $\mathrm{P}$ \\
\hline Periventricular & $3.1(1.6)$ & $3.9(1.6)$ & 3.880 & 0.051 \\
Deep white matter & $6.1(5.2)$ & $8.5(5.5)$ & 4.104 & 0.045 \\
Basal ganglia & $1.5(2.2)$ & $2.6(4.4)$ & 3.118 & 0.080 \\
Infratentorial & $0.4(1.1)$ & $0.6(1.9)$ & 0.505 & 0.479 \\
Total score & $11.0(8.2)$ & $15.6(9.6)$ & 5.853 & 0.017 \\
\hline
\end{tabular}

Values represent mean with standard deviation in parenthesis. Analyses were performed by analyses of covariance testing, controlling for age, presence or absence of hypertension and diabetes mellitus, low density lipoprotein-cholesterol and serum homocysteine levels. $\mathrm{OH}$ : orthostatic hypotension. women. The mean age at examination was $68.5 \pm 10.1$ years, and the mean duration of motor symptoms was $2.1 \pm 1.0$ years. The severities of parkinsonian motor symptoms were as follows: mean total UPDRS score was $24.7 \pm 17.9$ (part 1, 2.2 \pm 2.3 ; part 2, $7.2 \pm 5.8$; part 3, $14.8 \pm 10.7$ ). Twenty-nine patients had hypertension (HBP), 24 had diabetes mellitus (DM), and 106 were non-smokers. The presence or absence of hypertension and diabetes, and current/past smoking habits did not affect cerebral WMH (HBP vs. no HBP group: $14.9 \pm 8.6$ vs. $11.9 \pm 9.0, \mathrm{~F}=1.295, p=0.258 ; \mathrm{DM}$ vs. no DM group: $15.8 \pm$ 6.7 vs. $11.8 \pm 9.3, \mathrm{~F}=2.118, p=0.148$; non-smoker vs. exsmoker vs. current-smoker $12.9 \pm 8.9$ vs. $9.8 \pm 10.1$ vs. $11.7 \pm$

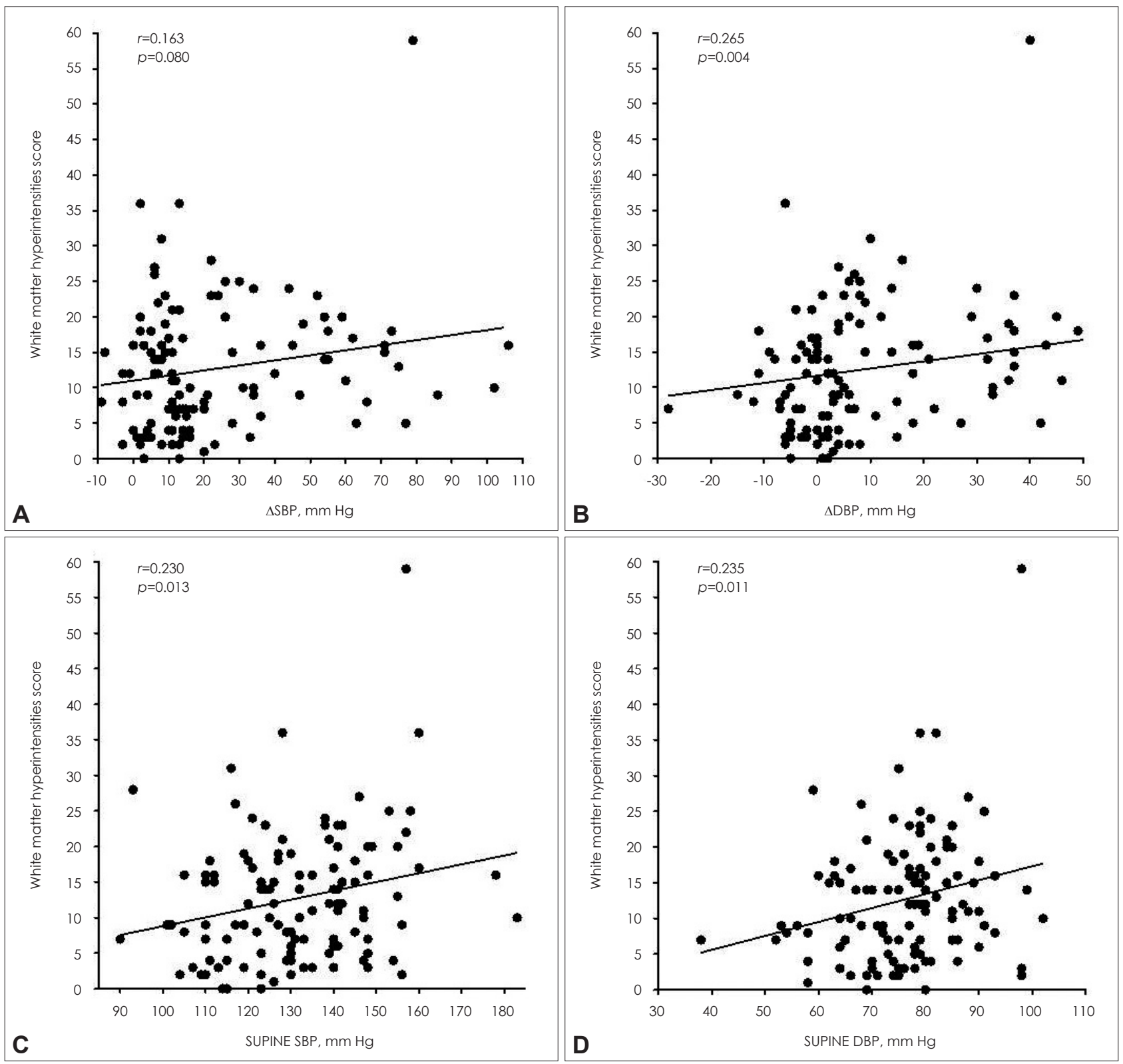

Figure 1. Scatter diagram of individual orthostatic blood pressure change (A and B) and supine blood pressure (C and D) and in association with white matter hyperintensities scores. The white matter changes were more severe along with orthostatic $\triangle \mathrm{DBP}$ and supine blood pressure increase in Spearman's correlation analysis. 
8.4, $\mathrm{F}=0.259, p=0.772$, by ANCOVA controlling for age, LDL-cholesterol and serum homocysteine). Higher LDL-cholesterol and serum homocysteine levels also did not correspond to increasing WMH scores $(r=0.004, p=0.965$ in LDL-cholesterol; $r=0.071, p=0.494$ in homocysteine by Spearman's rank correlation).

Clinical and laboratory profiles of the groups with and without orthostatic hypotension are presented in Table 1. Data from patients with and without orthostatic hypotension were similar for age, gender, duration of illness, severity of parkinsonian motor symptoms, and proportion of patients with hypertension and diabetes. Among the 42 patients with $\mathrm{OH}, 29$ were clinically symptomatic (69.1\%).

The WMH scores were higher in the orthostatic hypotension group compared to those in the non-OH group after controlling for age, the presence of hypertension and diabetes, LDL-cholesterol and serum homocysteine. In addition, a trend of more WMH lesions in the supratentorial region of the $\mathrm{OH}$ group was also found (Table 2). On correlation analysis, orthostatic diastolic blood pressure change was positively correlated with white matter changes (Figure 1A and B). On the other hand, supine blood pressures were closely correlated with the WMH scores of the whole brain (Figure 1C and D). When comparing groups with extremes of WMHs, the severe WMH group had higher supine systolic BP and larger fall in BP during orthostasis (Figure 2).

\section{Discussion}

The clinical importance of WMH has been emphasized fre- quently. Epidemiological investigations in healthy populations have shown that WMH predict the risk of cerebrovascular disease, cognitive dysfunction, and mortality. ${ }^{1}$ Advanced age, arterial hypertension, diabetes, and elevated serum homocysteine levels are risk factors for increasing $\mathrm{WMH} .^{2-4,14,15}$ However, these associations remain unclear in patients with PD. ${ }^{5,6}$ In this study, the presence or absence of cerebrovascular risk factors was not associated with WMH in patients with PD. This finding is consistent with previous studies and suggests that other nonvascular factors contribute to the vascular brain damage in patients with PD.

Orthostatic hypotension is common in patients with PD and appears more frequently as disease progresses and influence subjective symptoms, quality of life, and disease treatment. ${ }^{16}$ Recent investigations in patients with multiple system atrophy indicate that supine systolic blood pressure and/or blood pressure changes during orthostasis are significantly and independently correlated with WMH. ${ }^{17,18}$ However, a similar phenomenon was not confirmed in patients with PD. ${ }^{18}$ Prior studies were not performed in patients with early stage and the enrolled patients were not free from drug effects. In this study, we enrolled only newly diagnosed patients with early stage PD who had not taken any dopaminergic medication. Because dopaminergic drugs can aggravate orthostatic hypotension, this result suggests that this association was independent from anti-parkinsonian treatment.

We found that the orthostatic hypotension group had more severe WMH changes and that the differences in WMH were more significant in the deep white matter region. Severe orthostatic blood pressure drop was closely related to increasing

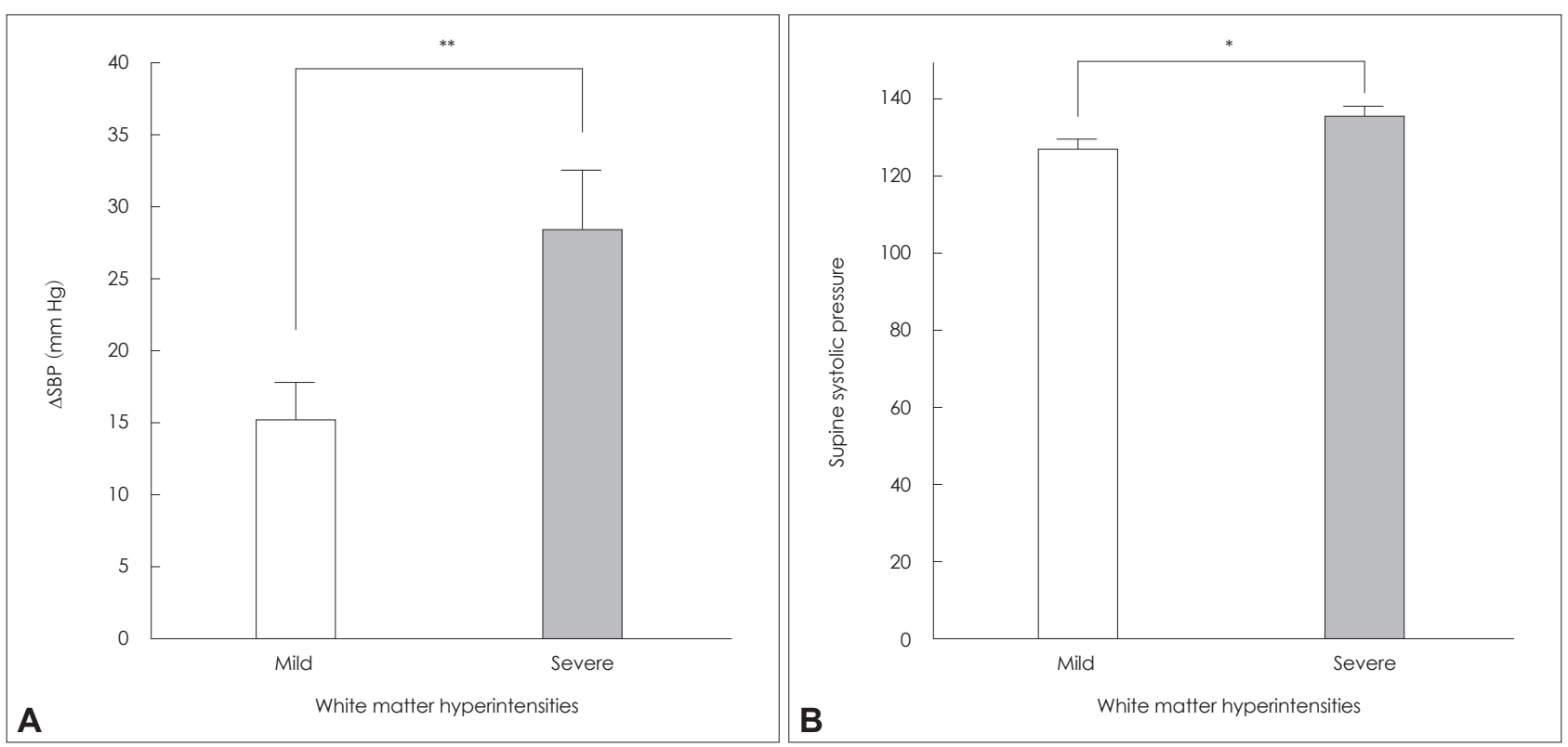

Figure 2. Mean values for blood pressure changes in patient groups stratified in terms of mild vs. severe white matter intensities (WMHs); (A) systolic blood pressure change ( $\triangle \mathrm{SBP}$ ) during tilt testing; and (B) supine systolic BP. The group with severe WMH had greater orthostatic fall in systolic BP, and higher supine systolic BP than did the group with relatively mild WMH. *Group difference significant, with $p<0.05,{ }^{* *} p<0.01$. Error bars indicate the standard error of the mean (SEM). 
WMH in tertile analysis. In addition, supine blood pressure may contribute in developing WMHs in PD.

Although the hemodynamic consequences during orthostasis have not been clarified, it was reported that systemic blood flow is inversely associated with WMHs in the elderly. ${ }^{19} \mathrm{Be}-$ cause orthostatic hypotension leads to recurrent blood pressure drops, this could result in cerebral hypoperfusion and impact neurocirculatory failures, perhaps via cerebral small-vessel disease. ${ }^{20}$ Supine blood pressures can also contribute myriad episodes of cerebral hypo- and hyper-perfusion to white matter injuries. In addition to neurocirculatory insufficiency, degenerative processes associated with aging and disease itself may contribute to the generation of WMH in PD.

In summary, our results indicate that hemodynamic changes associated with orthostatic hypotension may be associated with white matter changes in patients with PD. It provides important guideline for managing blood pressure in patients with PD.

\section{REFERENCES}

1. Debette S, Markus HS. The clinical importance of white matter hyperintensities on brain magnetic resonance imaging: systematic review and meta-analysis. BMJ 2010;341:c3666.

2. Hajjar I, Quach L, Yang F, Chaves PH, Newman AB, Mukamal K, et al. Hypertension, white matter hyperintensities, and concurrent impairments in mobility, cognition, and mood: the Cardiovascular Health Study. Circulation 2011;123:858-865.

3. de Leeuw FE, de Groot JC, Oudkerk M, Witteman JC, Hofman A, van Gijn J, et al. Hypertension and cerebral white matter lesions in a prospective cohort study. Brain 2002;125(Pt 4):765-772.

4. van Dijk EJ, Breteler MM, Schmidt R, Berger K, Nilsson LG, Oudkerk $\mathrm{M}$, et al. The association between blood pressure, hypertension, and cerebral white matter lesions: cardiovascular determinants of dementia study. Hypertension 2004;44:625-630.

5. Sławek J, Wieczorek D, Derejko M, Dubaniewicz M, Brockhuis B, Sitek E, et al. The influence of vascular risk factors and white matter hyperintensities on the degree of cognitive impairment in Parkinson's disease. Neurol Neurochir Pol 2008;42:505-512.

6. Lee SJ, Kim JS, Yoo JY, Song IU, Kim BS, Jung SL, et al. Influence of white matter hyperintensities on the cognition of patients with Parkinson disease. Alzheimer Dis Assoc Disord 2010;24:227-233.

7. Goldstein DS. Dysautonomia in Parkinson's disease: neurocardiological abnormalities. Lancet Neurol 2003;2:669-676.

8. Sharabi Y, Goldstein DS. Mechanisms of orthostatic hypotension and supine hypertension in Parkinson disease. J Neurol Sci 2011;310:123128.

9. Park A, Stacy M. Non-motor symptoms in Parkinson's disease. J Neurol 2009;256 Suppl 3:293-298.

10. Eguchi K, Kario K, Hoshide S, Hoshide Y, Ishikawa J, Morinari M, et al. Greater change of orthostatic blood pressure is related to silent cerebral infarct and cardiac overload in hypertensive subjects. Hypertens Res 2004;27:235-241.

11. Gibb WR, Lees AJ. The relevance of the Lewy body to the pathogenesis of idiopathic Parkinson's disease. J Neurol Neurosurg Psychiatry 1988;51:745-752.

12. Kaufmann H. Consensus statement on the definition of orthostatic hypotension, pure autonomic failure and multiple system atrophy. Clin Auton Res 1996;6:125-126.

13. Scheltens P, Barkhof F, Leys D, Pruvo JP, Nauta JJ, Vermersch P, et al. A semiquantative rating scale for the assessment of signal hyperintensities on magnetic resonance imaging. J Neurol Sci 1993;114:7-12.

14. Vermeer SE, van Dijk EJ, Koudstaal PJ, Oudkerk M, Hofman A, Clarke R, et al. Homocysteine, silent brain infarcts, and white matter lesions: The Rotterdam Scan Study. Ann Neurol 2002;51:285-289.

15. de Bresser J, Tiehuis AM, van den Berg E, Reijmer YD, Jongen C, Kappelle LJ, et al. Progression of cerebral atrophy and white matter hyperintensities in patients with type 2 diabetes. Diabetes Care 2010; 33:1309-1314.

16. Ziemssen T, Reichmann H. Cardiovascular autonomic dysfunction in Parkinson's disease. J Neurol Sci 2010;289:74-80.

17. Lim TS, Lee PH, Kim HS, Yong SW. White matter hyperintensities in patients with multiple system atrophy. J Neurol 2009;256:1663-1670.

18. Umoto M, Miwa H, Ando R, Kajimoto Y, Kondo T. White matter hyperintensities in patients with multiple system atrophy. Parkinsonism Relat Disord 2012;18:17-20.

19. Jefferson AL, Tate DF, Poppas A, Brickman AM, Paul RH, Gunstad J, et al. Lower cardiac output is associated with greater white matter hyperintensities in older adults with cardiovascular disease. J Am Geriatr Soc 2007;55:1044-1048.

20. Räihä I, Tarvonen S, Kurki T, Rajala T, Sourander L. Relationship between vascular factors and white matter low attenuation of the brain. Acta Neurol Scand 1993;87:286-289. 